04;09

\title{
Ударное сжатие лазерной плазмы \\ для увеличения интенсивности сигнала при спектрометрическом определении микрокомпонентов в рудах
}

\author{
(C) А.С. Закускин, А.М. Попов, Н.Б. Зоров, Т.А. Лабутин
}

Московский государственный университет им. М.В. Ломоносова, Москва,

Россия

`E-mail: timurla@laser.chem.msu.ru

Поступило в Редакцию 11 июля 2017 г.

Исследовано ударное сжатие лазерной плазмы при испарении образца руды на примере изменений интенсивности линий $\mathrm{Ag} \mathrm{I} 328.07 \mathrm{~nm}$ и Au I $267.60 \mathrm{~nm}$. Установлено, что максимальное увеличение интенсивности линий наблюдается на временах $2-2.5 \mu \mathrm{s}$, что также соответствует увеличению температуры плазмы до $5900 \mathrm{~K}$. Отношение сигнал/шум несколько ниже при использовании ударного сжатия плазмы, однако общий рост интенсивности сигнала позволяет увеличить чувствительность метода лазерно-искровой эмиссионной спектрометрии при определении микрокомпонентов в рудах.

DOI: 10.21883/PJTF.2018.02.45468.16964

Определение общего элементного состава руд, а также определение в них элементов с содержанием, не превышающим их средней распространенности в земной коре, являются важными современными задачами спектрального анализа. Для решения таких задач к аналитическим методам предъявляется ряд дополнительных требований, таких как экспрессность, низкая стоимость анализа, возможность проведения анализа без растворения пробы. Лазерно-искровая эмиссионная спектрометрия (ЛИЭС) является одним из наиболее перспективных методов прямого анализа, удовлетворяющих данным требованиям. Метод ЛИЭС основан на фокусировании мощного лазерного излучения на поверхности или в объеме образца. Под действием лазерного пучка происходит испарение небольшого количества вещества, сопровождающееся образованием 
плазмы. Ее излучение разлагается в спектр и используется для качественного и количественного анализа образца.

Этот простой и универсальный метод позволяет проводить прямой анализ практически всех химических элементов в твердых материалах, жидкостях и газах. Однако его чувствительности часто недостаточно для определения фоновых содержаний многих элементов в геологических материалах [1]. Для увеличения интенсивности сигнала могут применяться различные варианты дополнительного воздействия на мишень: использование двух или более лазерных импульсов в различных геометрических конфигурациях, резонансное возбуждение атомов [2] или основного компонента пробы [3], использование ударного сжатия плазмы. Последний способ не требует дополнительного оборудования и представляется весьма перспективным. Он позволяет существенно улучшить чувствительность ЛИЭС при выполнении измерений как на воздухе [4], так и водной среде [5].

Хорошо известно, что образование и расширение лазерно-индуцированной плазмы сопровождается образованием ударной волны [6,7]. Если на пути ударной волны располагается препятствие, волна отражается от этой преграды в сторону фронта плазмы. В результате столкновения во фронте плазмы увеличиваются температура, число возбужденных частиц и интенсивность эмиссионного сигнала. Таким образом, целью настоящей работы является использование пространственного сжатия плазмы для увеличения интенсивности ее свечения и соответственно чувствительности ЛИЭС.

Для выполнения измерений с ударным сжатием плазмы были разработаны и изготовлены микрокамеры цилиндрической формы. Микрокамера размещалась на столике для образца над поверхностью твердой пробы. Лазерное излучение фокусировалось через небольшое конусообразное отверстие в пространство микрокамеры. Излучение образующейся плазмы собиралось с торца микрокамеры через вертикальное отверстие шириной $0.85 \mathrm{~mm}$ и при помощи цилиндрической линзы направлялось на торец оптоволокна. В настоящей работе была использована камера диаметром $4 \mathrm{~mm}$ и высотой $4 \mathrm{~mm}$. Использовалась экспериментальная ЛИЭС-система, подробно описанная в [8]. Все измерения проводились при энергии единичного лазерного импульса $83 \mathrm{~mJ}$. Излучение, падающее на образец, фокусировалось на $6 \mathrm{~mm}$ ниже его поверхности во избежание пробоя на воздухе (диаметр пятна $\sim 0.3 \mathrm{~mm}$, плотность мощности $\sim 3 \mathrm{GW} / \mathrm{cm}^{2}$ ). Регистрация всех спек- 
Таблица 1. Параметры линий $\mathrm{Ag}, \mathrm{Au}, \mathrm{Fe}$, использующихся в работе

\begin{tabular}{|c|c|c|c|c|c|}
\hline \multicolumn{2}{|r|}{$\begin{array}{c}\text { Конфигурация и термы нижних } \\
\text { и верхних уровней переходов }\end{array}$} & $\begin{array}{l}\text { Длина } \\
\text { волны, }\end{array}$ & $\begin{array}{l}g_{k} A_{k i} \\
10^{7} \mathrm{~s}^{-1}\end{array}$ & $\begin{array}{c}\text { Elower } \\
\mathrm{eV}\end{array}$ & $\begin{array}{c}E_{\text {upper }}, \\
\mathrm{eV}\end{array}$ \\
\hline $\mathrm{Ag} \mathrm{I}$ & $\begin{array}{l}4 d^{10} 5 s-4 d^{10} 5 p \\
{ }^{2} S-{ }^{2} P^{0}\end{array}$ & 328.07 & 56 & 0 & 3.778 \\
\hline $\mathrm{Au} \mathrm{I}$ & $\begin{array}{c}5 d^{10} 6_{s}-5 d^{10} 6 p \\
{ }^{2} S-{ }^{2} P^{\mathrm{o}}\end{array}$ & 267.60 & 32.8 & 0 & 4.632 \\
\hline Fe I & $\begin{array}{c}3 d^{7}\left({ }^{2} D 2\right) 4 s-3 d^{6}\left({ }^{1} G 2\right) 4 s 4 p\left({ }^{3} P^{\mathrm{o}}\right) \\
a^{3} D-u^{3} F^{\mathrm{o}}\end{array}$ & 329.20 & 54.8 & 3.251 & 7.017 \\
\hline Fe I & $\begin{array}{c}3 d^{7}\left({ }^{4} P\right) 4_{s}-3 a^{5}\left({ }^{6} S\right) 4 s^{2} 4 p \\
a^{5} P-v^{5} P^{\mathrm{o}}\end{array}$ & 329.26 & 7.9 & 2.223 & 5.987 \\
\hline
\end{tabular}

тров проводилась при установленном стробе в $500 \mathrm{~ns}$, а каждый спектр был получен путем усреднения сигнала от 25 лазерных импульсов. В качестве мишени использовался стандартный образец медно-золотосеребряного концентрата OREAS 991 с содержанием золота и серебра $47.0 \pm 0.3$ и $48.1 \pm 0.9 \mathrm{ppm}$ соответственно.

В качестве эмиссионных линий, по которым возможно исследовать изменение интенсивности свечения плазмы в условиях ее ударного сжатия, были выбраны резонансные линии Ag I $328.07 \mathrm{~nm}$ и $\mathrm{Au} \mathrm{I}$ $267.60 \mathrm{~nm}$, параметры которых приведены в табл. 1 в соответствии с базой данных Куруца [9]. Сравнение характера изменений интенсивности свечения плазмы в условиях свободного расширения и ударного сжатия в микрокамере проводилось по пиковой интенсивности линий серебра и золота. На рис. 1 представлены зависимости интенсивностей этих линий от времени. Видно, что и для линии $\mathrm{Ag}$ I $328.07 \mathrm{~nm}(a)$, и для линии $\mathrm{Au}$ I $267.60 \mathrm{~nm}(b)$ при использовании микрокамеры наблюдается эффект увеличения интенсивности, максимум которого приходится на времена наблюдения около 2-2.5 $\mu \mathrm{s}$. Это, по-видимому, совпадает со временем столкновения отраженной ударной волны с фронтом плазмы в микрокамере с данными геометрическими параметрами. Затем происходит быстрый спад интенсивности, так что после $3.5-4 \mu \mathrm{s}$ она становится ниже, чем в случае свободно расширяющейся плазмы.

6 Письма в ЖТФ, 2018, том 44, вып. 2 

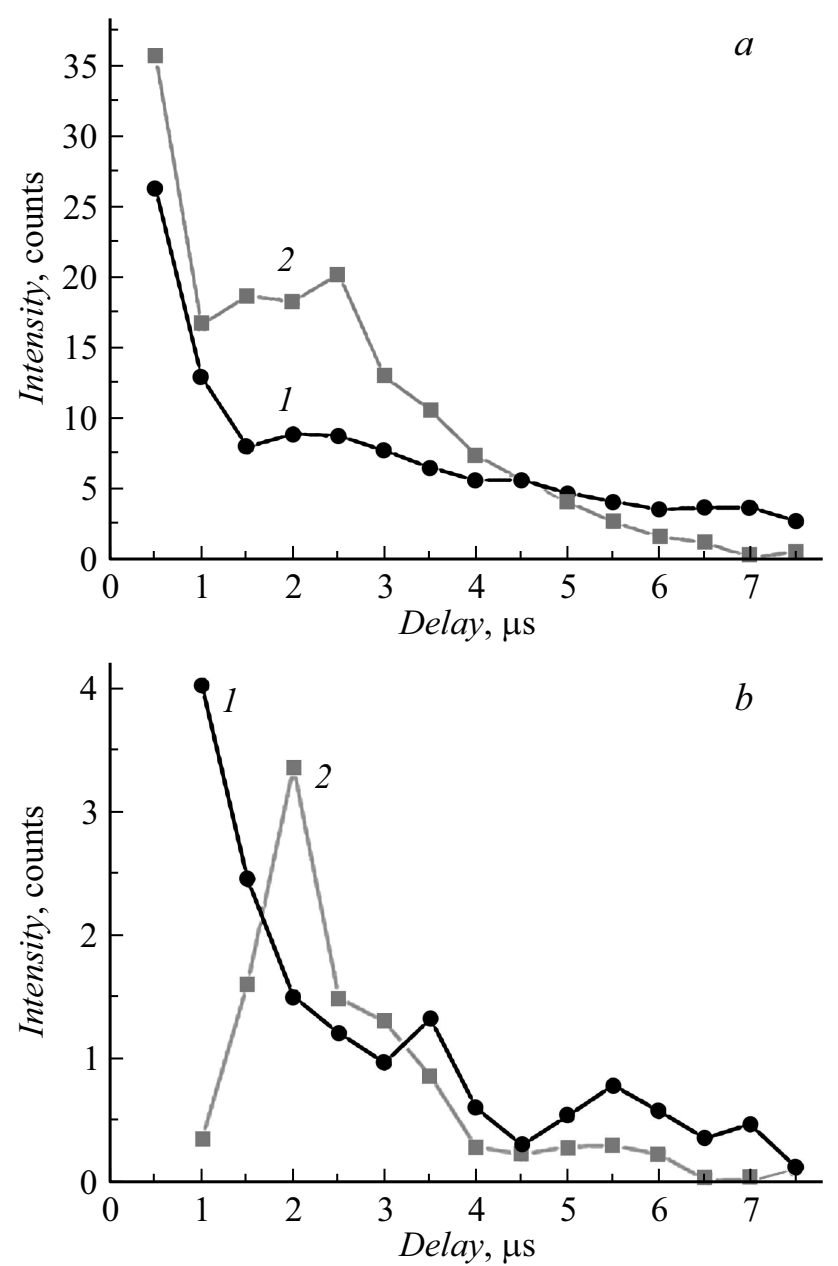

Рис. 1. Эволюция линий $\mathrm{Ag}$ I $328.07 \mathrm{~nm}(a)$ и $\mathrm{Au} \mathrm{I} 267.60 \mathrm{~nm}(b) .1$ - свободно расширяющаяся плазма, 2 - плазма в микрокамере.

Наблюдению выбранной линии золота препятствует ряд проблем, связанных с ее очень низкой интенсивностью. Сигнал оказывается слабым, даже несмотря на высокое содержание золота в образце OREAS

Письма в ЖТФ, 2018, том 44, вып. 2 

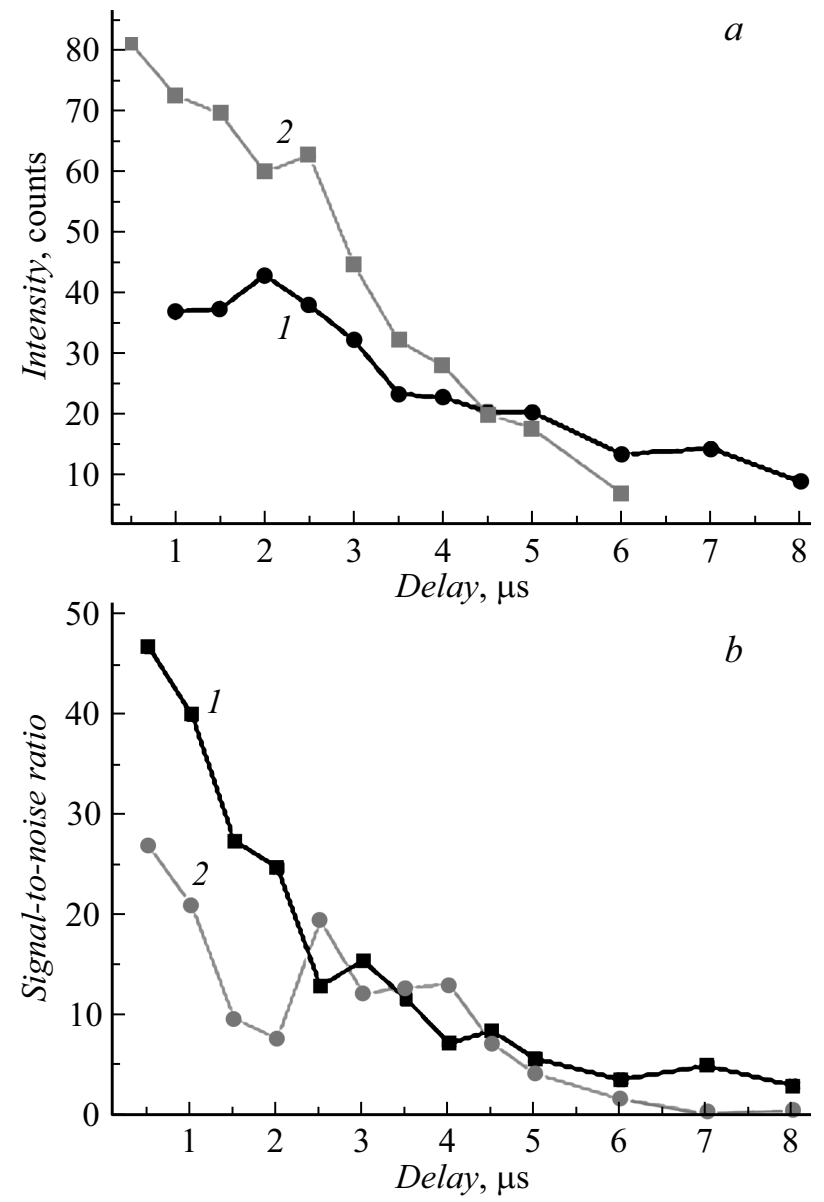

Рис. 2. Изменение интегральной интенсивности линии $\mathrm{Ag} \mathrm{I} 328.07 \mathrm{~nm}(a)$ и отношения сигнал/шум $(b) .1-$ свободно расширяющаяся плазма, $2-$ плазма в микрокамере.

991. Также из рис. 1 можно видеть, что пиковая интенсивность линии золота при использовании микрокамеры увеличивается приблизительно в 2 раза относительно варианта свободного расширения плазмы, тогда

$6^{*}$ Письма в ЖТФ, 2018, том 44, вып. 2 


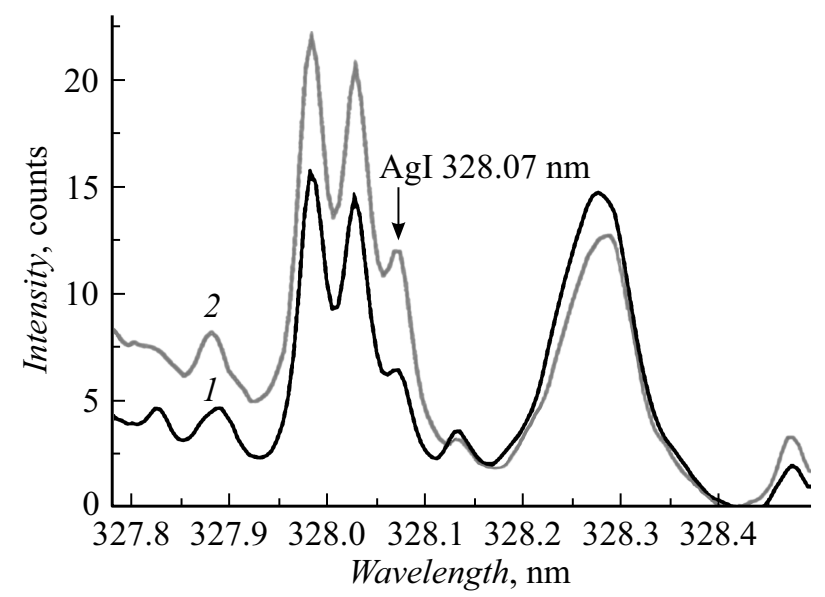

Рис. 3. Спектры свободно расширяющейся плазмы (1) и плазмы в микрокамеpe $(2)$.

как для линии серебра наблюдается более чем троекратное увеличение. Несмотря на то что изменение интенсивности линии Au I $267.60 \mathrm{~nm}$ наглядно отражает характер изменения интенсивности свечения плазмы при ее ударном сжатии, интенсивность сигнала остается недостаточной (сопоставимой с уровнем шумов) для определения золота в рудах методом ЛИЭС.

Вблизи линии серебра $\mathrm{Ag}$ I $328.07 \mathrm{~nm}$ имеется ряд спектральных помех, усложняющих корректное определение ее интенсивности [10]. B частности, наблюдается ее частичное перекрывание с линией Fe I $328.03 \mathrm{~nm}$. В связи с этим для более правильного определения интенсивности линии серебра целесообразно использовать ее интегральный сигнал после деконволюции сигналов с аппроксимацией контуров линий функцией Лоренца. На рис. 2, $а$ представлена зависимость интегральной интенсивности линии серебра Ag I $328.07 \mathrm{~nm}$ от времени наблюдения в случае использования микрокамеры и при свободном расширении плазмы. Как видно, на временах до $4 \mu \mathrm{s}$ происходит существенное увеличение интегрального сигнала при использовании пространственного сжатия плазмы. По-прежнему локальный максимум наблюдается вблизи задержки в $2.5 \mu \mathrm{s}$, т. е. сразу после столкновения фронта ударной волны

Письма в ЖТФ, 2018, том 44, вып. 2 
Таблица 2. Температура плазмы

\begin{tabular}{c|c|c}
\multirow{2}{*}{} & \multicolumn{2}{|c}{$T, \mathrm{~K}$} \\
\cline { 2 - 3 } & $2.5 \mu \mathrm{s}$ & $4.5 \mu \mathrm{s}$ \\
\cline { 2 - 3 } Без микрокамеры & 5000 & 4800 \\
С микрокамерой & 5900 & 4800
\end{tabular}

с плазмой. Необходимо отметить, что общее увеличение интенсивности линий при использовании микрокамеры позволяет упростить процедуру деконволюции и уменьшить погрешность их аппроксимации.

Поскольку основной интерес представляет не столько общее повышение интенсивности эмиссионного сигнала плазмы, сколько улучшение чувствительности определения отдельных элементов, нами было рассмотрено отношение сигнала линии Ag I $328.07 \mathrm{~nm}$ к шуму. Данный параметр может оказывать большое влияние на воспроизводимость результатов определения, а в литературе приводятся данные о том, что при ударном сжатии плазмы уровень шумов в спектре может увеличиваться до 1.5 раз по сравнению со случаем свободно расширяющейся плазмы [11]. Расчет отношения сигнал/шум проводился для интегрального сигнала линии серебра, поскольку, как было показано выше, в этом случае следовало ожидать более значимого улучшения. За уровень шума мы принимали стандартное отклонение фонового сигнала на небольшом участке спектра, лежащем вблизи линии серебра и не имеющем спектральных помех. На рис. 2, $b$ представлена зависимость отношения сигнал/шум от времени наблюдения. Действительно, при использовании микрокамеры на большей части временно́й шкалы флуктуации сигнала от импульса к импульсу увеличиваются. Однако при задержке $2.5 \mu \mathrm{s}$, когда наблюдается наибольшее относительное увеличение интенсивности линии серебра в микрокамере, отношение сигнал/шум оказывается выше, чем при свободном расширении плазмы. Также увеличение интенсивности за счет ударного сжатия плазмы более значимо, чем негативный эффект от повышения уровня шумов в спектре, поскольку влияние спектральных помех на линию серебра гораздо больше, чем зашумленности спектра вблизи нее.

Для того чтобы сделать предположения о механизме увеличения интенсивности эмиссионного сигнала при ударном сжатии плазмы,

Письма в ЖТФ, 2018, том 44, вып. 2 
нами была оценена температура плазмы по методу двух линий [12]. Для этого использовались линии Fe I $329.20 \mathrm{~nm}$ и $\mathrm{Fe}$ I $329.26 \mathrm{~nm}$, параметры которых приведены в табл. 1. В табл. 2 представлены результаты определения температуры в момент времени, соответствующий максимальному усилению линии $\mathrm{Ag}$ I $328.07 \mathrm{~nm}(2.5 \mu \mathrm{s})$, и в момент $4.5 \mu \mathrm{s}$, когда интенсивности линий при использовании микрокамеры и без нее оказываются одинаковыми. Можно видеть, что в первом случае температура плазмы в микрокамере оказалась на $900 \mathrm{~K}$ выше, чем в случае, когда микрокамера не использовалась, а равные интенсивности линии серебра наблюдаются при равных температурах плазмы. По-видимому, прохождение фронта ударной волны приводит к дополнительному разогреву плазмы, увеличению степени атомизации вещества и числа возбужденных частиц в ней, а следовательно, и к росту эмиссионного сигнала.

Таким образом, при ударном сжатии лазерной плазмы наблюдается увеличение общей интенсивности ее свечения, что можно наблюдать на примере линии $\mathrm{Ag} \mathrm{I} 328.07 \mathrm{~nm}$ (рис. 3). Проведение измерений в момент времени, соответствующий максимальному увеличению сигнала и отношения сигнал/шум, может значительно улучшить чувствительность определения микрокомпонентов в рудах методом ЛИЭС.

Работа выполнена при поддержке РФФИ (грант № 15-33-70053мол_а_мос).

\section{Список литературы}

[1] Harmon R.S., Russo R.E., Hark R.R. // Spectrochim. Acta B. 2013. V. 87. P. 11-26.

[2] Shen X.K., Wang H., Xie Z.Q., Gao Y., Ling H., Lu Y.F. // Appl. Opt. 2009. V. 48. N 13. P. 2551-2558.

[3] Goueguel C., Laville S., Vidal F., Sabsabi M., Chaker M. // J. Anal. Atom. Spectrom. 2010. V. 25. N 5. P. 635-644.

[4] Wang Y., Chen A., Sui L., Li S., Liu D., Wang X., Jiang Y., Huang X., Jin M. // Phys. Plasmas. 2016. V. 23. N 11. P. 113105.

[5] Lazic V., Laserna J.J., Jovicevic S. // Spectrochim. Acta B. 2013. V. 82. P. 50-59.

[6] Гарбарук А.В., Гричкевич М.С., Калмыков С.Г., Можсаров А.М., Петренко М.В., Сасин М.Э. // Письма в ЖТФ. 2014. Т. 40. В. 21. С. 97-103.

[7] Гарбарук А., Грищкевич М.С., Калмыков С.Г., Сасин М.Э. // Письма в ЖТФ. 2016. T. 42. B. 19. C. $38-45$.

Письма в ЖТФ, 2018, том 44, вып. 2 
[8] Zaytsev S.M., Popov A.M., Zorov N.B., Labutin T.A. // J. Instrum. 2014. V. 9. P. 06010.

[9] Kurucz's atomic line database from CD-ROM N 23. Available on web: http://www.cfa.harvard.edu/amp/ampdata/kurucz23/sekur.html

[10] Popov A.M., Labutin T.A., Zaytsev S.M., Seliverstova I.V., Zorov N.B., Kal'ko I.A., Sidorina Y.N., Bugaev I.A., Nikolaev Y.N. // J. Anal. Atom. Spectrom. 2014. V. 29. N 10. P. 1925-1933.

[11] Popov A.M., Colao F., Fantoni R. // J. Anal. Atom. Spectrom. 2010. V. 25. N 6. P. 837-848.

[12] Aragon C., Aguilera J.A. // Spectrochim. Acta B. 2008. V. 63. P. 893-916. 